\title{
Mechanical Design of a Second Generation LHC IR Quadrupole
}

\author{
S. Caspi, S.E. Bartlett, D.R. Dietderich, P. Ferracin, S.A. Gourlay, R.R. Hafalia, C.R. Hannaford, A.F. \\ Lietzke, A.D. McInturff, G. Sabbi, and R.M. Scanlan
}

\begin{abstract}
One of the proposed options to increase the LHC luminosity is the replacement of the existing inner triplets at the Interaction Regions with new low-beta larger aperture quadrupoles operating at the same gradient. Lawrence Berkeley National Laboratory (LBNL) is carrying out preliminary studies of a large-bore $\mathrm{Nb}_{3} \mathrm{Sn}$ quadrupole. The mechanical design presents a support structure based on the use of keys and bladders without self-supporting collars. This technology has been proven effective in several successful common coil $\mathrm{Nb}_{3} \mathrm{Sn}$ dipoles built at LBNL, and it is for the first time applied to a $\cos (2 \vartheta)$ design. In this paper we present a detailed analysis of the quadrupole mechanical behavior, demonstrating the possibility of delivering, through this method, well-controlled coil precompression during assembly, cool-down and excitation. The study has been performed with the finite element program ANSYS.
\end{abstract}

Index Terms - $\mathrm{Nb}_{3} \mathrm{Sn}$, Large Hadron Collider, Quadrupole, Superconducting Magnets.

\section{INTRODUCTION}

$\mathrm{I}_{\mathrm{c}}^{\mathrm{n}}$ n a recent LHC Accelerator Research Program (LARP) collaboration meeting [1], the USA has taken the first collaborative effort in looking at the LHC Interaction Region (IR) beyond the current design. Three US laboratories are currently engaged in the magnet program in collaboration with CERN: LBNL [2], [3], FNAL [4], [5], and BNL [6], and it appears that a variety of unique high field dipoles and quadrupoles will have to be considered to accomplish the desired goals. At the present time, the principle design challenge is the design of a single bore IR quadrupole. Operational demands on these quadrupoles are expected to be severe: a reliable high operating field-gradient $(205 \mathrm{~T} / \mathrm{m})$ throughout a large bore $(90 \mathrm{~mm})$, with excellent field quality, and high radiation load. $\mathrm{Nb}_{3} \mathrm{Sn}$ superconducting cable appears inevitable and a long-range program may incorporate high $\mathrm{T}_{\mathrm{c}}$ conductor. Simultaneous achievement of these goals requires a mechanical support structure that will immobilize the conductor against large accumulated Lorentz loads

Manuscript received October 20, 2003. This work was supported under contract DE-AD03-76SF00098 by the Director, Office of Energy Research, Office of High Energy Physics, U.S. Department of Energy.

All authors are with Lawrence Berkeley National Laboratory, Berkeley, CA 94720 (phone: 001510486 7244; fax: 001510486 5310; e-mail: s_caspi@lbl.gov).
( $\sim 100 \mathrm{MPa})$, without exceeding the maximum $\mathrm{Nb}_{3} \mathrm{Sn}$ stressdegradation limit (150MPa) during magnet assembly. Traditional self-supporting collars are believed to be inadequate for this task, due to large pre-stress losses from collaring "spring-back" and cool-down.

This paper summarizes the latest design study of the bladder and key coil pre-stressing and support system that has been successfully used in the $\mathrm{Nb}_{3} \mathrm{Sn}$ high-field dipole-magnet program at LBNL [7], [8]. This system uses bladders for lowcost, precisely controlled pre-stressing during magnet assembly. The insertion of interference keys retains the prestress and allows for removal of the bladders. The use of a tensioned aluminum shell to compress internal iron components causes a substantial fraction of the operational pre-stress to be accumulated during cool-down, allowing the operational pre-stress to be approached from below, without substantial danger of overshooting. Satisfactory assembly and cool-down mechanical performance must be demonstrated before submitting brittle coils to their influence. Accordingly, a complete 3D structural analysis has been carried out to establish the efficacy, and/or limitations, of the basic concept. This paper reports the results of this analysis

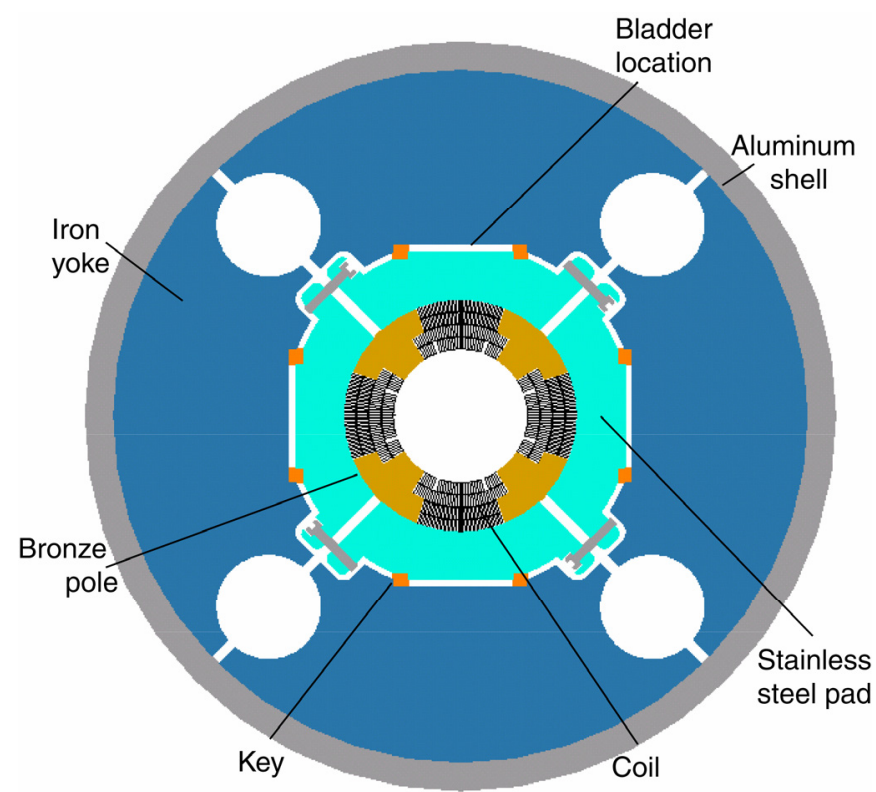

Fig. 1. Magnet cross-section: coils, stainless steel pads, iron yokes and outer aluminum shell. Keys in the interference gap transmit the compression forces from the yoke to the coil pads, and allow the pre-stressing bladders to be removed for operation. 


\section{MAGNET DESIGN}

The magnet cross-section in Fig. 1 shows a four-layer, $\cos (2 \vartheta)$ coil system, with a $90 \mathrm{~mm}$ bore. The coil cross-section and associated field quality have been discussed elsewhere [2], [3]. These coils are positioned and supported within a quadrupole-symmetric pre-stressing and coil-support structure. Compressive mechanical forces, generated by the outer aluminum shell tension, are concentrated onto the coils through a set of iron yokes and stainless-steel pads, whose separation is maintained by interference keys (Fig. 1). During magnet assembly, pressurized bladders establish the gap between the pads and yokes, simultaneously pre-compressing the coils, pads and yokes. The resulting tension in the outer shell is easily monitored with strain gauges and compared to the bladder pressure. Keys are inserted into the resulting gap, to allow the bladders to be deflated and removed, preparatory to cool-down. The difference in thermal expansion between the various material properties causes a substantial increase in coil pre-stress during cool-down. This allows a significant reduction in coil stress during assembly.

When the coil is pre-stressed enough so that the pole-turn does not separate from the pole during excitation, the increase in the shell's tension will be approximately constant, resulting in extremely small conductor displacements.

In the axial direction (parallel to the magnet's bore), prestress is also required to withstand the Lorentz forces generated by the coil ends. In the present design (Fig. 2) four aluminum tubes within the yoke's cooling holes are connected to end plates, which bear against the coil's end-shoes. As with the radial pre-stress, the initial axial pre-stress is augmented during cool-down to reduce axial coil displacements.

\section{3D STRESS ANALYSIS}

\section{A. Creating the SOLID model}

Creating an accurate full 3D structural model required the integration of several computer programs, including $\operatorname{ProE}^{\circledR}$ (CAD), ANSYS ${ }^{\circledR}$ (thermal-stress-strain) and TOSCA $^{\circledR}$ (magnetic field). To simplify the mechanical analysis, the 4layer coil cross-section (previously optimized with $\mathrm{ROXIE}^{\circledR}$ ), was modeled as a magnetically equivalent 2-layer coil, whose end windings were generated with a modified version of the program "BEND". The inner layer included a single wedge and end spacer; the outer layer had none. The coordinates of all turns were converted into a ProE trail file, loaded into ProE as solids, and treated as single-coil blocks. They were then extended to fill the entire radial space between fixed inner and outer cylindrical surfaces. Spacers, pole pieces and end shoes were assembled between coil-blocks to create a contiguous cylindrical body, and the remained structural components (pads, yoke and shell) were added around the outer coil.

\section{B. The 3D mechanical model (ANSYS)}

An ANSYS translator was used to convert the ProE solid model into an ANSYS volume model. An ANSYS input file was executed, to identify and name individual components of the solid model, and assign material properties and meshing requirements. Elements were generated (Fig. 3) using a volume sweep of 20-node structural element (SOLID95). Assembly components were not glued. They were allowed to interact via contact elements (TARGE170 and CONTA174) along adjacent surfaces.

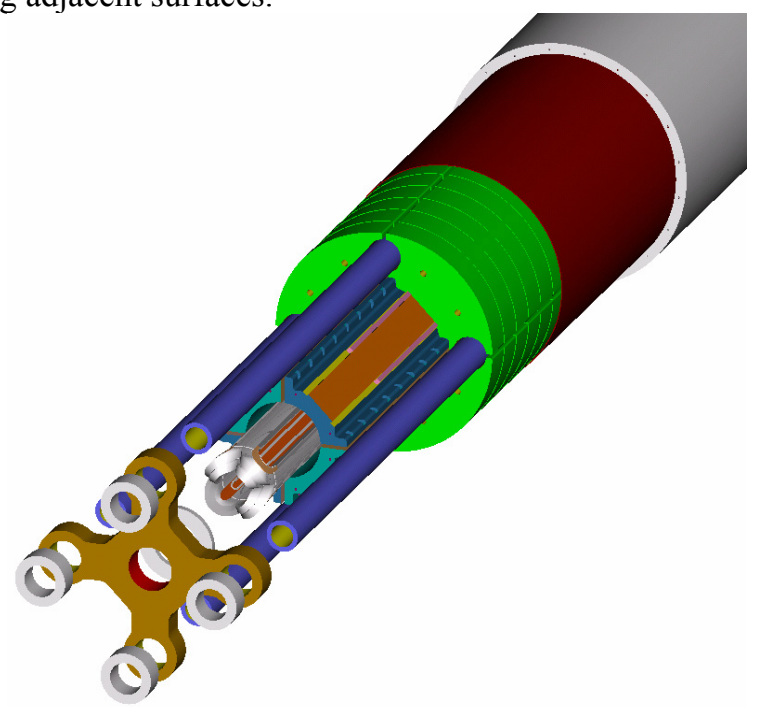

Fig. 2. Exploded view of the end region, showing how end plate, axial tie rods, coil and pads assembly, yokes, and shell are modeled.

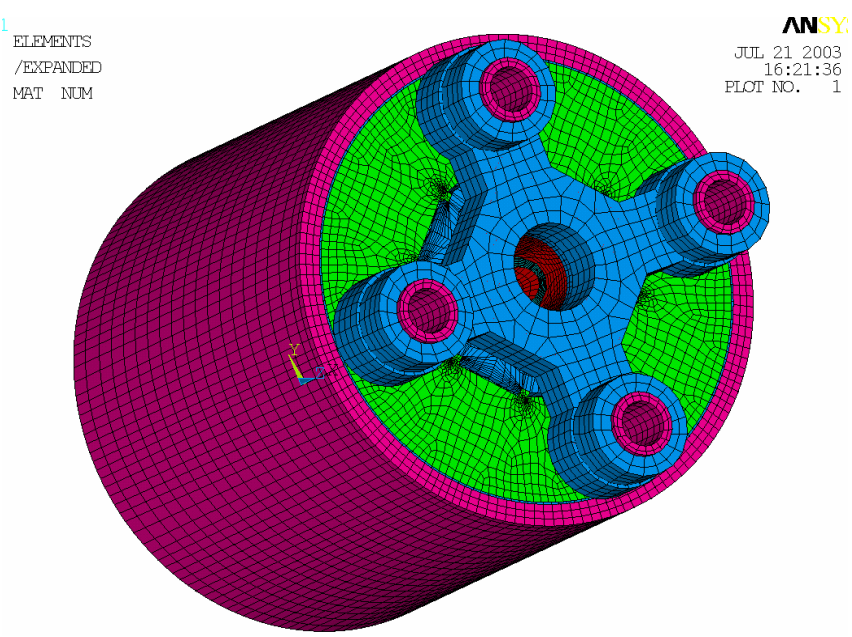

Fig. 3. The 3D ANSYS meshed model of the quadrupole, showing one endplate, the yokes and the shell.

The entire conductor/structure loading process was simulated by the following four steps. 1) Bladder pre-stressing: uniform pressures were applied to all bladder-contact areas of the pads and yokes. The keys were "turned off", allowing them to respond to geometric changes, but preventing them from transmitting forces. 2) Keying: the interference keys were "turned on", and the bladder pressure deleted, using the ANSYS "alive" operation. 3) Cool-down to $4.2 \mathrm{~K}$ : the temperature of all solids was changed from $300 \mathrm{~K}$ to $4.2 \mathrm{~K} .4$ ) Magnetic excitation: instead of computing Lorentz forces in ANSYS, which would have required a new model, the Lorentz forces were calculated with TOSCA.

\section{The 3D magnetic model (TOSCA)}

Creating the model within TOSCA required only the coils and iron yokes, thereby reducing the problem size (by excluding unneeded components like poles, shoes, pads and 
shell). The individual turns of the initial coil geometry were read into TOSCA from an input file as 8-node conductorelement bricks (Fig. 4). The assigned current was 20kA/turn, corresponding to a gradient of $270 \mathrm{~T} / \mathrm{m}$.

\section{Transferring Lorentz forces from TOSCA to ANSYS}

A Lorentz force density map was created in TOSCA, and converted to a finite force/element within ANSYS, that was applied proportionally to each element's nodes. This procedure [9], executed as an ANSYS input file, created a file containing the coordinates of the geometric center of each meshed conductor element. TOSCA assigned the vector J x B, force density cross-product, to each coordinate, producing a map read by ANSYS. The force density was scaled by the volume of each conductor element, and proportionately distributed to the element's nodes.

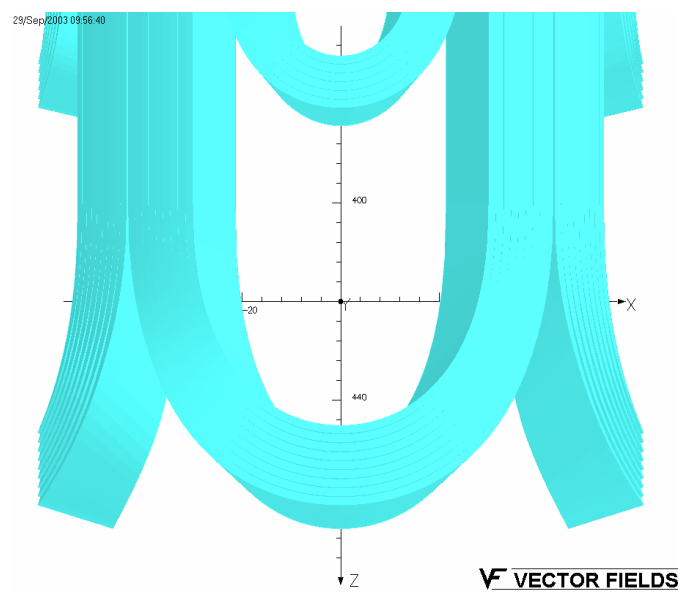

Fig. 4. Inner layer end modeled in TOSCA from 8-node conductor brick elements.

\section{Calculation Results}

\section{A. Assembly, Cool-down and Excitation}

Following cool-down (step 3), TOSCA-computed Lorentz force-densities, consistent with the proposed cable's short sample current (20kA), were scaled linearly in ANSYS to simulate Lorentz forces above and below the short sample limit. The absolute principal-stress in the shell, and the coil's inner layer pole and mid-plane turns are plotted in Fig. 5, from assembly through excitation.

During assembly, pre-stressing was stopped with $50 \mathrm{MPa}$ of tension in the shell. While this relaxed to $45 \mathrm{MPa}$ onto the keys, it increased to $155 \mathrm{MPa}$ during cool-down. The shell's tension is azimuthally uniform, except for some curvature changes near the iron gaps. The inner coil's azimuthal stress peaks at $50 \mathrm{MPa}$ during assembly, increasing to $120 \mathrm{MPa}$ with cool-down.

With Lorentz excitation, the stress in the shell is nearly constant, while the stress in the coil varies linearly with the square of the current. Pressure at the pole declines, while pressure at the mid-plane increases. At short sample ( $\mathrm{I} / \mathrm{Iss}=1)$ inner layer's pole and mid-plane stresses are $15 \mathrm{MPa}$ and $150 \mathrm{MPa}$ respectively. Slightly beyond short sample, the coil separates from the winding pole-island, after which, the shell tension is observed to increase rapidly with excitation.

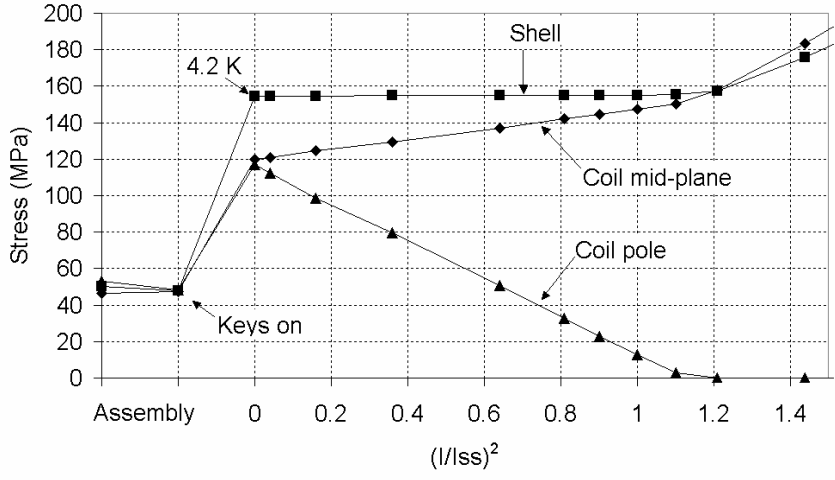

Fig. 5. The absolute stress in the shell (tension), and in the inner layer's pole and mid-plane turns (compression), during assembly, cool-down and excitation. The normalized current has been extended beyond short sample to illustrate the impact of allowing separation from the pole.

\section{B. Ends}

Analysis of the magnet ends proved to be particularly interesting and helped determine the desired pre-load from the aluminum axial end-rods. The analysis points out the existence of two undesirable end effects. The first is the separation of the pole turn from the winding island tip. The second is the axial sliding between the pole-turn and the island along the straight section. Although the coil's end-plate and rods have been introduced to reduce axial conductor displacements during operation, Lorentz forces are sufficiently strong to overcome a reasonable axial pre-stress in the ends. Further axial pre-loading may over-compress the coil's straight section near the end, potentially causing the cable to de-cable.
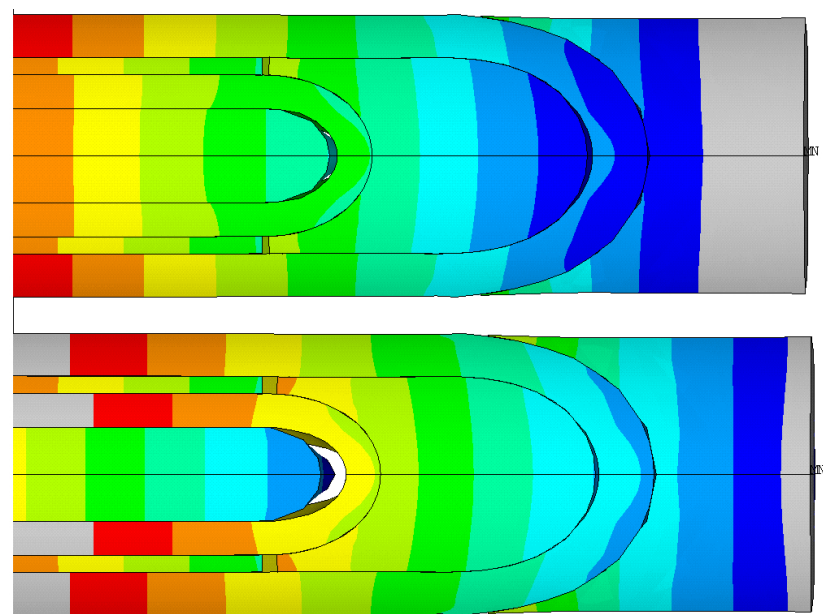

Fig. 6. Exaggerated relative axial displacements of inner layer's end-region at short sample excitation: no friction (bottom) and 0.3 friction factor (top) between coil and island.

The calculated frictionless displacements of the inner layer's end turns (Fig. 6, bottom) revealed separation from the poleisland. Before excitation, the inner layer's straight-section stress was $120 \mathrm{MPa}$, azimuthal compression, and $45 \mathrm{MPa}$, axial compression. When a coefficient friction $(\mu=0.3)$ is introduced between the pole-turn and the island, the predicted relative displacement between coil and island reduced (Fig. 6, top). A plot of the relative axial displacement between the pole-turn 
and the island from assembly through excitation (Fig. 7) revealed that frictionless slipping started around $40 \%$ of Iss, and monotonically accumulated to $3 \mathrm{~mm}$ at short sample $(\mathrm{I} / \mathrm{Iss}=1)$. With some friction $(\mu=0.3)$, this decreased to $0.05 \mathrm{~mm}$

Sliding during excitation results from the simultaneous increase in the axial end Lorentz force, and reduction in the transverse compression of the pole-turn against the island. The present results suggest that an increase in the azimuthal precompression, with a sufficiently large friction factor between coil and island may be required to eliminate sliding with the assumed axial loading.

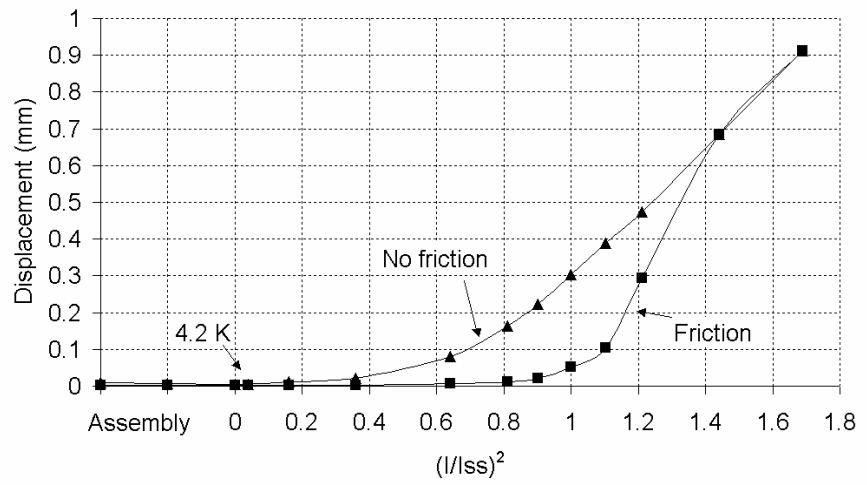

Fig. 7. Relative displacements between inner layer pole-turn and the island, for two friction factors $(\mu=0$, and $\mu=0.3)$.

\section{FUTURE PLANS}

The quadrupole support structure has been fabricated (Fig.8), and is approaching the final assembly. Several experiments are planned. The structure will be assembled for mechanical testing, around a $90 \mathrm{~mm}$ diameter, split, aluminum tube (Fig. 9). The stress relationship between the inner tube and the outer shell will be measured under a variety of bladder pressures, key sizes and temperatures. The radial displacements of the inner wall will also be measured and correlated to assembly tolerance variations.

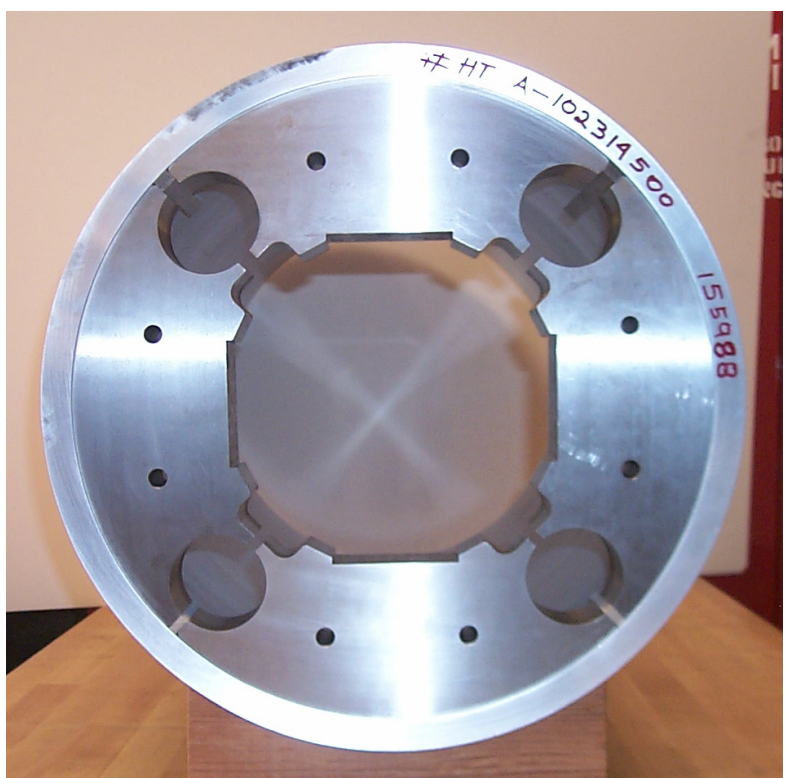

Fig. 8. A $22 \mathrm{~mm}$ thick, $500 \mathrm{~mm}$ diameter aluminum shell mounted around a four way split iron yoke.

$\mathrm{Nb}_{3} \mathrm{Sn}$ coil testing is planned in two stages. In the first stage, a double-layer coil, similar to the outer quad coil, will be built, using existing tooling from the D20 dipole project. After successful testing, a second phase will commence, with fabrication and testing of an added inner double-layer coil.

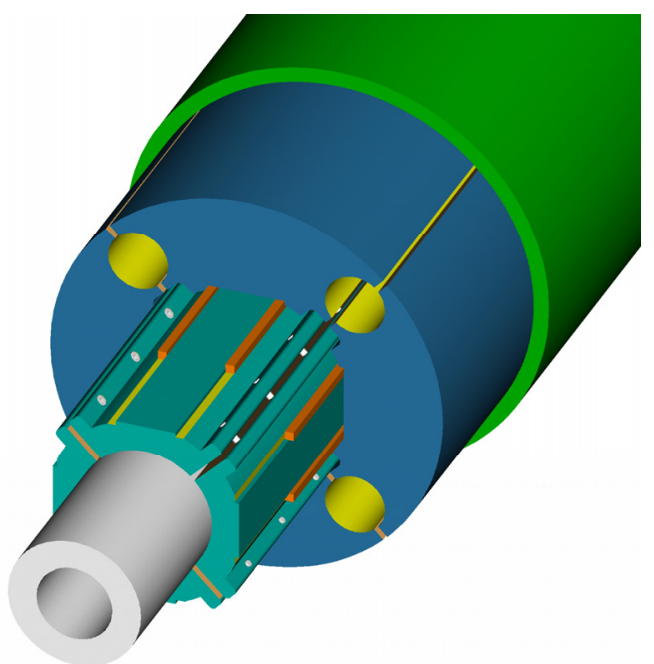

Fig. 9. A 3D model of the planned mechanical structure test, using $90 \mathrm{~mm}$ diameter aluminum tube in place of expensive, fragile coils.

\section{CONCLUSION}

The mechanical structure of the next generation high field quadrupole magnet has been analyzed in $3 \mathrm{D}$, from assembly, through cool-down, and Lorentz loading. A key and bladder assembly system is proposed to pre-stress the system inside an outer aluminum shell. This will allow a low assembly prestress, yet be sufficient to prevent azimuthal coil separation from the island, due to the substantial pre-stress gain obtained during cool-down,. Sufficient pole-turn friction against the pole-island is required to eliminate axial slippage of the poleturn relative to the island

\section{REFERENCES}

[1] LARP Collaboration meeting, http://www.agsrhichome.bnl.gov/LARP/ Port Jefferson, September 16-18, 2003.

[2] G. Sabbi et al., "Nb 3 Sn quadrupole magnets for the LHC IR", IEEE Trans. Appl. Supercond., . Vol. 13, no. 2, June 2003, pp. 1262-1265.

[3] P. Ferracin, et al., "Field quality analysis of the next Generation IR Quadrupole for the LHC", presented at 2004 Particle Accelerator Conference, Portland, OR, May 2003.

[4] A. Zlobin et al., "Conceptual design study of $\mathrm{Nb}_{3} \mathrm{Sn}$ low-beta quadrupole for $2^{\text {nd }}$ generation LHC IRs", IEEE Trans. Appl. Supercond., Vol. 13, no. 2, June 2003, pp. 1266-1269.

[5] A. Zlobin, et al., "Aperture Limitations for 2nd Generation Nb3Sn LHC IR Quadrupoles", presented at 2004 Particle Accelerator Conference, Portland, OR, May 2003.

[6] R. Gupta, at al., "Next generation IR magnets for hadron colliders", IEEE Trans. Appl. Supercond., Vol. 13, no. 2, June 2003, pp. 13511354

[7] R.R. Hafalia et al., "A new support structure for high field magnet", IEEE Trans. Appl. Supercond., vol. 12, no. 1, March 2002, pp. 47-50.

[8] S. Caspi et al., "The use of pressurized bladders for stress control of superconducting magnets", IEEE Trans. Appl. Supercond., vol. 11, no. 1, March 2001, pp. 2272-2275. 
[9] S. Prestemon, Lawrence Berkeley National Lab., Berkeley, CA, private communication, February 2003. 